\title{
Autophagy-related protein 12 associates with anti-apoptotic B cell lymphoma-2 to promote apoptosis in gentamicin-induced inner ear hair cell loss
}

\author{
XIAOFENG MA ${ }^{1-3^{*}}$, YONGZE LIU ${ }^{2,3 *}$, WAQAS MUHAMMAD ${ }^{4}$, DINGDING LIU ${ }^{2}$, \\ JUNGUO WANG $^{2}, \mathrm{HAN}_{\mathrm{ZHOU}}^{2}, \mathrm{XIA} \mathrm{GAO}^{1-3}$ and XIAOYUN QIAN ${ }^{2,3}$ \\ ${ }^{1}$ Department of Otorhinolaryngology-Head and Neck Surgery, Nanjing Drum Tower Hospital, \\ Clinical College of Nanjing Medical University; ${ }^{2}$ Department of Otorhinolaryngology-Head and Neck Surgery, \\ Affiliated Drum Tower Hospital of Nanjing University Medical School, Jiangsu Provincial Key Medical Discipline \\ (Laboratory); ${ }^{3}$ Research Institution of Otorhinolaryngology, Nanjing, Jiangsu $210008 ;{ }^{4}$ State Key Laboratory \\ of Bioelectronics, Institute of Life Sciences, Southeast University, Nanjing, Jiangsu 210096, P.R. China
}

Received January 24, 2016; Accepted February 15, 2017

DOI: $10.3892 / \mathrm{mmr} .2017 .6458$

\begin{abstract}
The aim of the present study was to investigate the underlying mechanisms of autophagy in a gentamicin (GM)-induced ototoxic model, and to establish whether the blocking of autophagy significantly increases the survival of inner ear hair cells. Cochleae were carefully dissected from four day-old C57BL/6J mice and randomly divided into three groups prior to explant culture: Control (culture medium), GM-treated (culture medium + GM) and GM + 3-methyladenine (3-MA; culture medium + GM + 3-MA). Transmission electron microscopy, immunofluorescence and western blotting were performed to observe the expression of the autophagy protein microtubule-associated protein 1A/B-light chain 3 in explant cultures treated with GM and the autophagy inhibitor 3-MA. Administration of GM in in vitro mouse cochlear culture induced apoptosis and the formation of autophagic vesicles and autophagosomes in hair cells. Notably, combined treatment with GM and 3-MA to block autophagy significantly increased the survival of inner ear hair cells. Furthermore, it was indicated that the simultaneous expression and interaction of Atg12 with Bcl-2 following GM treatment co-integrated autophagy with apoptosis in the cochlea. The results of the present study demonstrated that autophagy was involved in
\end{abstract}

Correspondence to: Professor Xia Gao or Professor XiaoYun Qian, Department of Otorhinolaryngology-Head and Neck Surgery, Affiliated Drum Tower Hospital of Nanjing University Medical School, Jiangsu Provincial Key Medical Discipline (Laboratory), 321 Zhongshan Road, Nanjing, Jiangsu 210008, P.R. China

E-mail: xiagaogao@hotmail.com

E-mail: qxy522@163.com

*Contributed equally

Key words: autophagy, gentamicin, ototoxicity, cochlea
GM-induced ototoxicity. Additionally, Atg12 may serve a protective role by binding to $\mathrm{Bcl}-2$. Therefore, Atg12 may be a potential therapeutic target for the treatment of GM-induced cochlear hair loss.

\section{Introduction}

Aminoglycosides are renowned antibiotics primarily used in clinics for the treatment of tuberculosis, and effectively cure infections caused by aerobic gram-negative bacteria (1-3). Gentamicin (GM) is a widely-used aminoglycoside antibiotic that has attracted increasing attention recently due to its potent ototoxic side effects. Typically, aminoglycoside antibiotics are absorbed and accumulated in the inner ear lymph (4-6); hair cells of the inner ear have a high affinity for them. Previous studies have demonstrated in an ototoxicity model of GM that death of hair cells is attributed to apoptosis (7), and apoptosis is closely associated with autophagy (8).

Autophagy is a series of complex process within the cell where the components or foreign invaders are digested. It is an important underlying mechanism that maintains the steady and resistant adversity of the cells, and regulates dynamic alterations in cell membrane structure following lysosome-mediated degradation of cellular proteins and organelles (9-12). Autophagy is considered to be a general response to stress contributing to cell death; alternatively, it may be a cell cytoprotective mechanism $(13,14)$. Autophagy formation is comprised of the following four processes: Induction and formation of the pre-autophagosome structure, autophagosome formation, fusion with lysosomes and degradation (15). Homologous proteins that serve important roles in autophagy have additionally been identified in mammalian cells $(12,16)$, including microtubule-associated protein 1A/B-light chain 3 (LC3), the mammalian ortholog of yeast autophagy-related protein 8 (Atg8). The conjugation of LC3 to lipid phosphatidylethanolamine to form LC3-II, located in the autophagy body membrane, reflects the level of autophagy $(17,18)$. To date, 34 different types of Atg protein have been determined 
in yeast (19), which serve a key role in formation of the autophagosome.

Depending on the experimental conditions, autophagy may directly induce cell death or act as an underlying mechanism for cytoprotection. Despite recent rapid progress in autophagy research, little is understood about autophagy in the organ of Corti in GM-induced cochleotoxicity.

Cochleotoxicity induced by GM is a typical condition of the cochlea that generates stress, leading to inner ear hair cell death and tissue damage. To assess whether autophagy occurs in GM-induced cochlear injury and whether it contributes to inner ear hair cell loss or survival, the present study used a GM ototoxic experimental model to examine the underlying mechanisms. Apoptosis has been reported to contribute to cochleotoxicity $(7,20,21)$; however, the interactions between autophagy and apoptosis to induce cochlear pathology remain unclear. Autophagy is suppressed by Bcl-2 and related antiapoptotic family members, by binding to the $\mathrm{B}$ cell lymphoma-2 (Bcl-2) homology 3 (BH3) domain of Beclin-1, which is an essential component of the class III phosphatidyl inositol-3-kinase/Vps34 complex necessary for autophagy induction. The present study demonstrated that Atg12 interaction with Bcl-2 links autophagy and apoptosis during GM treatment of inner ear hair cells.

\section{Materials and methods}

Materials. GM sulfate (G1272) was purchased from Sigma-Aldrich; Merck KGaA (Darmstadt, Germany); C57BL/6J mice were acquired from the Model Animal Research Center of Nanjing University (Nanjing, China); 3-methyladenine (3-MA) was purchased from Sigma-Aldrich; Merck Millipore (M9281) and Dulbecco's modified Eagle's medium (DMEM) was obtained from Gibco; Thermo Fisher Scientific, Waltham, MA, USA. The following primary antibodies were used: Rabbit anti-Atg12 (2011S) and rabbit anti-Bcl-2 (50E3) from Cell Signaling Technology, Inc. (Danvers, MA, USA); rabbit anti-LC3 (L7543; Sigma-Aldrich; Merck Millipore) and mouse anti-Bcl-2 (SC-7382; Santa Cruz Biotechnology, Inc., Dallas, TX, USA). The following secondary antibodies were used: Cyanine (Cy3)-conjugated donkey anti-rabbit (A31572; Thermo Fisher Scientific, Inc.), horseradish peroxidase (HRP)-conjugated goat anti-rabbit (111-035-003; Jackson ImmunoResearch Laboratories, Inc., Westgrove, PA, USA) and IgG HRP-conjugated goat anti-mouse (SC-2005; Santa Cruz Biotechnology, Inc.). Fluorescein isothyanate (FITC)-phalloidin (P5282) and DAPI (D9542) were purchased from Sigma-Aldrich; Merck Millipore, and a Pierce Classic Immunoprecipitation kit was obtained from Thermo Fisher Scientific, Inc. (26146).

Dissection and culturing organ of Corti explants. A total of 160 C57BL/6J mice (age, 4 days; male:female, 1:1; weight, 3-5 g) were purchased from the Model Animal Research Center of Nanjing University (Nanjing, China). Mice were housed under a 12 -h light-dark cycle at $22 \pm 1^{\circ} \mathrm{C}$ and with a humidity of $56 \pm 5 \%$, with 5 mice per cage. Mice received an ad libitum $0.3 \%$ sodium diet. The care and use of animals were in strict accordance with the 'Guiding Directive for Humane treatment of Laboratory Animals' issued by the
Chinese National Ministry of Science and Technology. The Institutional Animal Care and Use Committee of Nanjing Medical University and Nanjing Drum Tower Hospital provided ethical approval for all procedures. All efforts were made to minimize the number of animals used and to prevent their suffering. Dissection and culturing was performed as previously described (22). Following cleaning with $75 \%$ ethanol, pups were decapitated and the cochleae were carefully dissected. Soft tissues and the cartilage were removed, and the cochlear epithelium was isolated. The organotypic cultures were maintained in serum-free media consisting of DMEM supplemented with glucose and $30 \mathrm{U} / \mathrm{ml}$ penicillin $\mathrm{G}$ (Sigma-Aldrich; Merck $\mathrm{KGaA}$ ) at $37^{\circ} \mathrm{C}$ in a humidified atmosphere containing $5 \% \mathrm{CO}_{2}$. After $24 \mathrm{~h}$, the culture medium was removed and replaced with fresh media containing $100 \mu \mathrm{m} / \mathrm{l} \mathrm{GM}$ or $10 \mathrm{mmol} / \mathrm{l} 3-\mathrm{MA}$ and incubated at $37^{\circ} \mathrm{C}$ for $24 \mathrm{~h}$. Cochlear explants were randomly divided into three groups: Control (culture medium), GM-treated (culture medium + GM) and GM- and 3-MA-treated (culture medium + GM + 3-MA).

Immunocytochemical analysis. A total of $48 \mathrm{~h}$ after dissection, the organ of Corti were immediately processed for immunofluorescent staining. Cochlear explants were fixed with $4 \%$ paraformaldehyde for $30 \mathrm{~min}$. Following fixation, they were rinsed with $0.1 \mathrm{mM}$ phosphate-buffered saline (PBS) three times and blocked with 5\% normal horse serum (Sigma-Aldrich; Merck KGaA) in PBS (pH 7.4) with $0.1 \%$ Triton X-100 for $1 \mathrm{~h}$ at room temperature. The tissues were incubated with an anti-LC3 primary antibody overnight at $4^{\circ} \mathrm{C}$. The next day the tissues were washed three times with $0.1 \%$ Tween in PBS, followed by incubation with the secondary Cy3-conjugated donkey anti-rabbit antibody for $2 \mathrm{~h}$ at room temperature. Following this, FITC-phalloidin and DAPI were added to label hair cell stereocilia and the nucleus, respectively. Following immunostaining, samples were mounted in Southern Biotech Fluoromount- $\mathrm{G}^{\mathrm{TM}}$ Slide Mounting medium (Thermo Fisher Scientific, Inc.). Cochleae were dissected into apical, middle and basal turns, and were imaged using a Nikon confocal fluorescence microscope (TE2000-U; Nikon Corporation, Tokyo, Japan).

Cytocochleograms and quantification of cochlear hair cells. For HC quantification in the GM-treated and GM + 3-MA-treated samples, the entire cochlea was imaged using a quantified analytic system for cochlea hair cells as previously described $(23,24)$. Missing inner hair cells (IHCs) and outer hair cells (OHCs) were counted over 0.24-mm intervals under a light microscope (magnification, x400). Cochleograms were generated demonstrating the percentage hair cell loss as a function of percentage distance from the apex. Negative controls were included to determine the amount of background staining. The Cochlea Anatomy Labshell Program (supplied as a gift from Professor Dalian Ding, Buffalo University, Buffalo, NY, USA) was used to compute the percentage of missing hair cells in $10 \%$ intervals along the length of the cochlea, starting from the apex. For each cochlea, the mean density of $\mathrm{HCs}$ was determined (apical, 10-20\% distance from the cochlear apex; middle, 40-50\% distance from the cochlear apex; basal, $70-80 \%$ distance from the cochlear apex). 
A

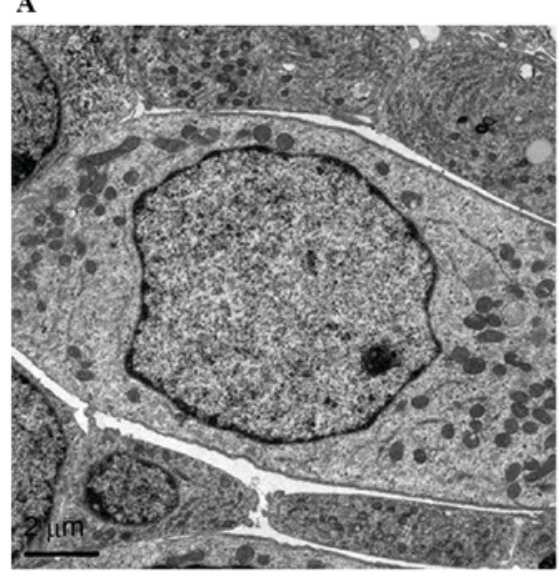

Control
B

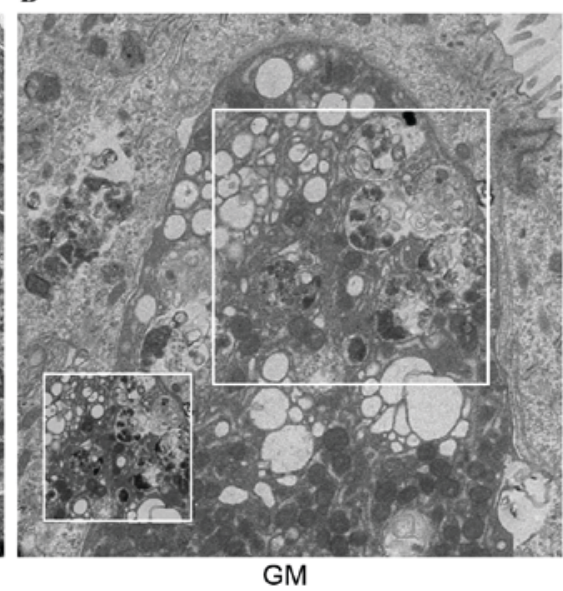

Figure 1. Autophagy in cochlea hair cells observed under a transmission electron microscope. (A) In the control group, the structure of cell membrane was intact, the stereocillia were arranged in order, and the organelles appeared healthy. (B) Following GM treatment, a large number of autophagosomes appeared inside the sensory epithelium and cell membrane was ruptured. Scale bar $=2 \mu \mathrm{m}$. GM, gentamicin.

Transmission electron microscopy (TEM) and scanning electron microscopy (SEM). Cochlea were collected after $48 \mathrm{~h}$ and fixed in $2.5 \%$ glutaraldehyde in $0.1 \mathrm{M}$ PBS at $4^{\circ} \mathrm{C}$ overnight. The samples were subsequently post-fixed in $1 \%$ osmium tetroxide and further processed by standard procedures, including dehydration, infiltration and polymerization in araldite. Ultrathin sections were post-stained and examined using a H-7650 transmission electron microscope (Hitachi, Ltd., Tokyo, Japan) (25). For SEM, the fixation procedure was performed as described above, following which samples were dehydrated in ethanol, critical point dried with a vacuum and coated with gold-palladium. The samples were observed by SEM for morphological description and for potential acoustic trauma assessment. Samples were imaged under a Hitachi S-3000 N Scanning Electron Microscope (Hitachi, Ltd.).

Western blot analysis and co-immunoprecipitation assay. Western blot analysis was performed to assess the protein expression levels of LC3 and Bcl-2 from cochlear explants following exposure to the various experimental conditions as described above. The cochleas were lysed with ice-cold radioimmunoprecipitation assay buffer (PP109; Protein Biotechnology, Beijing, China), containing Phosphatase Inhibitor Cocktail (Roche, Basel, Switzerland), to obtain the tissue proteins. Protein concentrations were measured using a bicinchoninic acid Protein Quantification kit (PP202, Protein Biotechnology) according to the manufacturer's protocol, using GAPDH (KC-5G4, 1:2,000; KangChen Biotech Inc., Shanghai, China) as the reference protein. Equivalent amounts of tissue lysate protein $(20 \mu \mathrm{g})$ were separated by gel electrophoresis on a 4-20\% gradient SDS-PAGE gel and transferred onto polyvinylidene fluoride membranes. Blots were blocked with $5 \%$ non-fat milk and then incubated with anti-LC3 $(1: 1,000)$ and anti-Bcl-2 (1:1,000) rabbit polyclonal antibodies at $4^{\circ} \mathrm{C}$ overnight. Membranes were then washed with $0.1 \%$ Tween 20 in PBS and probed with an HRP-conjugated goat anti-rabbit $(1: 5,000)$ secondary antibody for $2 \mathrm{~h}$ at room temperature. The bound antibody complexes were detected using an ECL Plus kit (Pierce; Thermo Fisher Scientific, Inc.) and a Storm 840
PhosphorImager system (Molecular Devices, LLC, Sunnyvale, CA, USA).

For endogenous co-immunoprecipitation, a rabbit monoclonal anti-Atg12 antibody was used. Precleared tissue lysates were incubated with anti-Atg12 at $4^{\circ} \mathrm{C}$ overnight, followed by incubation with protein $\mathrm{G}$ agarose beads for $1 \mathrm{~h}$ at room temperature. Beads were washed with lysis buffer and boiled in sample buffer. Western blot detection of Bcl-2 was performed with mouse anti-Bcl-2 $(1: 1,000)$ at $4^{\circ} \mathrm{C}$ overnight. Membranes were then washed with $0.1 \%$ Tween-20 in PBS and probed with an HRP-conjugated goat anti-mouse $(1: 5,000)$ secondary antibody for $2 \mathrm{~h}$ at room temperature. The bound antibody complexes were detected using an ECL Plus kit using a Storm 840 PhosphorImager system.

Statistical analysis. The data were presented as the mean \pm standard deviation. All experiments were repeated at least three times. Statistical analyses were conducted using Microsoft Excel 2007 (Microsoft Corporation, Redmund, WA, USA) and GraphPad Prism 5 (GraphPad Software, Inc., La Jolla, CA, USA). P $<0.05$ was considered to indicate a statistically significant difference.

\section{Results}

TEM analysis of autophagy in GM. GM-induced autophagy in the cochlea was evaluated by TEM, and the morphology of hair cells in GM-treated cochlea was investigated. As presented by representative micrographs, compared with the control (Fig. 1A), GM-treated cells (Fig. 1B) had autophagosomes with characteristic double or multiple membranes after $24 \mathrm{~h}$. In addition, numerous autophagic vacuoles or vesicles were observed.

Protective role of 3-MA on GM-induced hair cell loss. In order to assess cochleotoxicity and the protective underlying mechanism of 3-MA, cells were double-stained with FITC-phalloidin (green) to label hair cells, and DAPI (blue) as a nuclear stain (Fig. 2A). Previously, cochlear cultures from mice were incubated in medium containing $10 \mathrm{mmol} / \mathrm{l} 3-\mathrm{MA}$ and $0.1 \mathrm{mM} \mathrm{GM}$ for $24 \mathrm{~h}$. Loss of hair cells indirectly reflects 
A

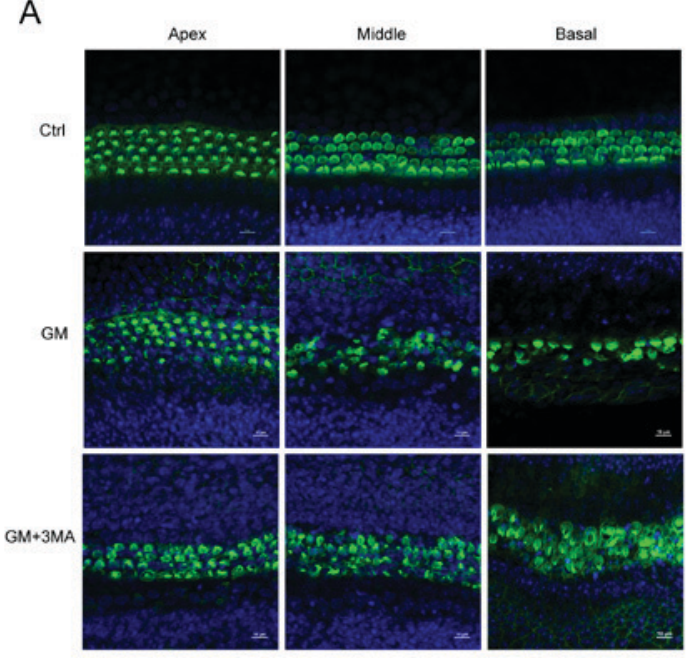

B
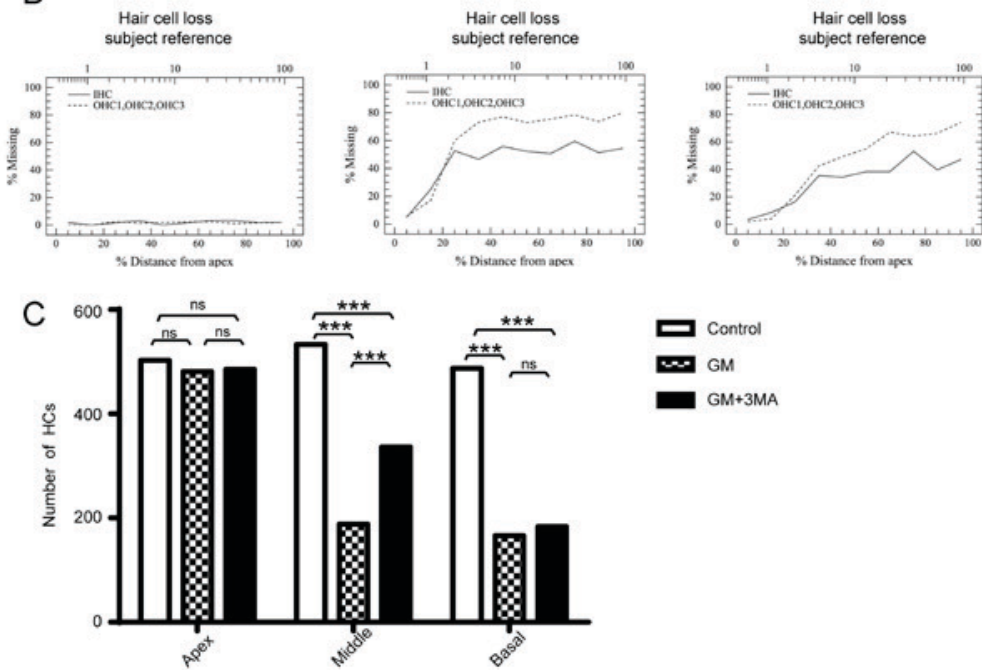

Figure 2. Morphological and quantitative analysis of hair cells in the different groups. (A) Explant cultures treated with GM, GM + 3-MA and control (no drug) stained with fluorescein isothyanate-phalloidin and DAPI exhibited variations in loss of hair cells in the apical, middle and basal regions. (B) Cytocochleograms representing the percentage of $\mathrm{IHC}$ and $\mathrm{OHC}$ loss as a percentage of total distance from the apex of the cochlea (n=6/group). (C) Hair cell density in the apical, middle and basal regions of the cultured cochlea treated with $100 \mu \mathrm{M} \mathrm{GM}$ alone or $100 \mu \mathrm{M} \mathrm{GM}+10 \mathrm{mM}$ 3-MA, compared with non-treated controls. OHC and IHC counts were significantly decreased in the middle region of the cochlea. Data are presented as the mean \pm standard deviation, all experiments were repeated at least three times. ${ }^{* * *} \mathrm{P}<0.001$. GM, gentamicin; IHC, inner hair cell; OHC, outer hair cell; 3-MA, 3-methyladenine; HCs, hair cells; Ctrl, control; ns, not significant.

A

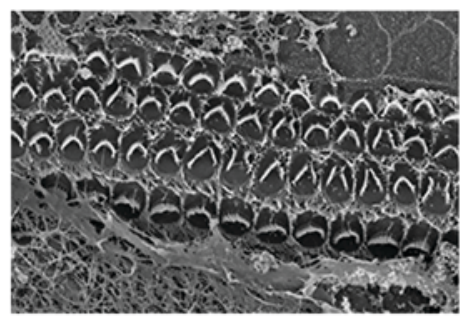

Control
B

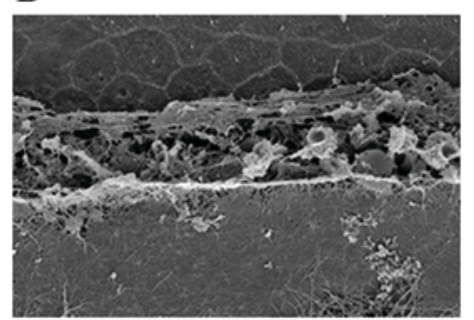

GM

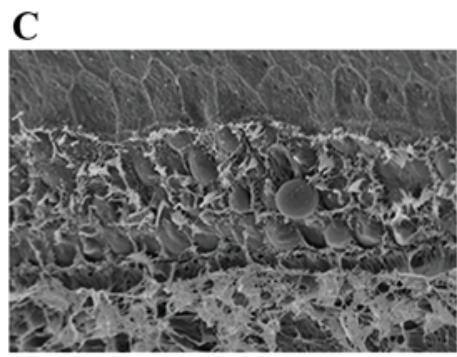

GM+3-MA

Figure 3. Scanning electron microscopy to assess cochlear surface structure. (A) The control group had three rows of OHCs and one row of IHCs. (B) The GM-induced ototoxic group had loss of numerous IHCs and OHCs, and the stereocilia of these cells were fused or completely absent. (C) Treatment with 3-MA preserved the majority of hair cells; however, not the stereocilia. 3-MA, 3-methyladenine; IHC, inner hair cell; OHC, outer hair cell.

cochleotoxicity; percentage hair cell loss was increased in the GM-treated group compared with the control group. However, the addition of 3-MA attenuated this effect, particularly in the middle region of the cochlea (Fig. 2B). Compared with untreated controls, GM severely distorted the anatomy of the organ of Corti in the GM-treated group, where the hair cells were deformed and disordered, and gaps were clearly observed. Following 3-MA treatment, the number and alignment of cells in the organ of Corti was improved compared with the GM group. FITC-phalloidin-labeled hair cells were counted in each group after $24 \mathrm{~h}$. No significant differences were observed in the number of hair cells in the apex between groups. Conversely, in the GM-treated group, OHC and IHC counts were significantly decreased in the middle region of the cochlea. However, in GM + 3-MA-treated group, 3-MA significantly protected against $\mathrm{OHC}$ and IHC loss in the middle region of the cochlea. In summary, these results suggested that 3-MA treatment significantly protects against GM-induced hair cell loss in the middle region of the cochlea.
SEM examination of the cochlear surface structure. SEM was performed to examine the surface structure of the cochlea. Examination of the organ of Corti of the control group (Fig. 3A) indicated three rows of OHCs and one row of IHCs. Conversely, cochleae from the GM-induced ototoxic group (Fig. 3B) exhibited loss of the well-known architecture of hair cells of the organ of Corti. The stereocilia of the OHCs and IHCs were disrupted, disarrayed, fused, had focal loss or were completely absent. Treatment with 3-MA preserved the majority of hair cells; however, the stereocilia became more dispersed (Fig. 3C).

Upregulation of LC3 protein expression levels following GM-induced injury. To examine whether GM injury affects protein expression levels of LC3, immunostaining and western blotting were performed on each group. LC3 was detected in the control and GM-treated groups; however, expression levels were markedly upregulated in the GM-treated group (Fig. 4A). In western blots, LC3 protein presents with double bands; one 
A

Basal
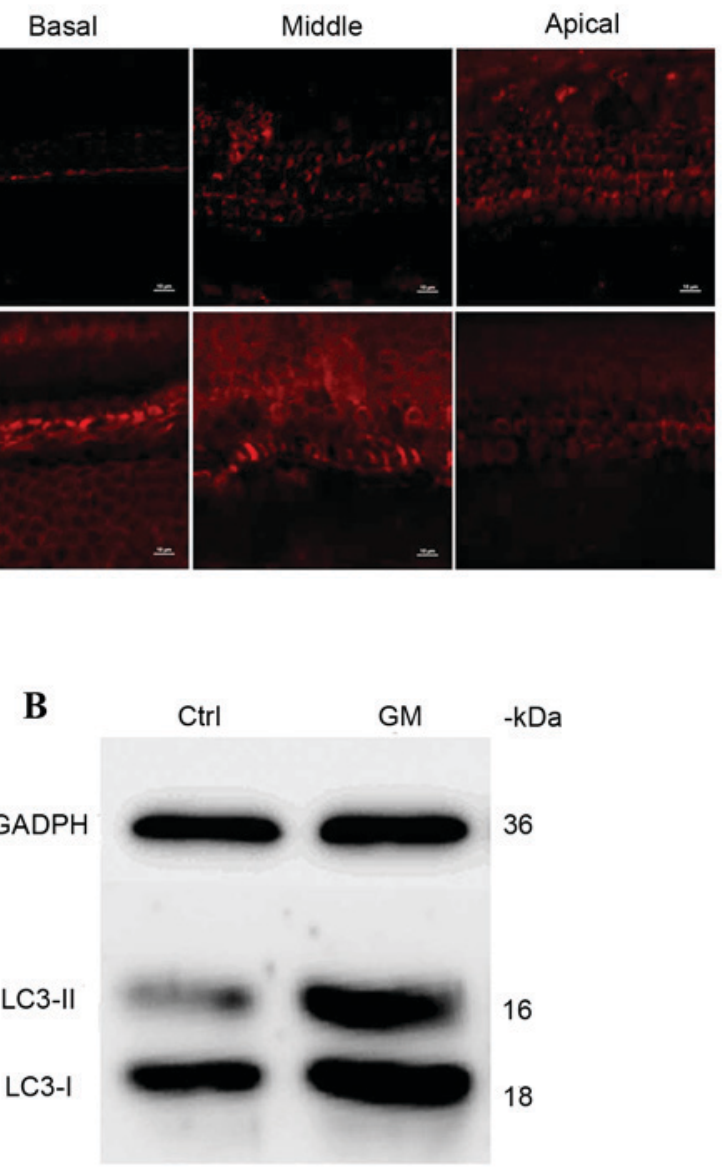

Figure 4. Protein expression levels of LC3. (A) Representative immunofluorescence microscopic images of LC3 demonstrating the comparable expression in the apical, middle and basal regions of explant cultures. (B) Representative western blot images of LC3 and LC3-II protein expression levels following GM treatment. GAPDH served as a protein loading control $(n=20)$. GM, gentamicin; LC3, microtubule-associated protein 1 light chain 3; Ctrl, control.

is unconjugated and the other is conjugated with phosphotidylethanolamine to form the LC3-II complex. This is recruited to the autophagosome membrane; increased expression levels reflects the level of autophagy in cells. LC3 protein levels were overexpressed in the GM-treated group compared with the control group (Fig. 4B).

Interaction of Atg12 with Bcl-2 to promote apoptosis in $G M$-treated cochleae. To assess the interaction between Atg12 and Bcl-2 in cochleae, western blotting was performed to observe protein expression levels of Bcl-2 in the GM-, $\mathrm{GM}+3$-MA-treated and control groups. Bcl-2 was equally expressed in all three groups (Fig. 5A). Co-immunoprecipitation was performed using a rabbit anti-Atg12 antibody to observe the protein complexes involved in ototoxicity and further analyze the interaction between Atg12 and Bcl-2 (Fig. 5B). Bcl-2 and Atg12 were co-expressed in the GM-treated and control groups, indicating that they interact in the cochlea. However, when autophagy was inhibited by $3-\mathrm{MA}, \mathrm{Bcl}-2$ protein expression levels were reduced, demonstrating that 3-MA interrupts the Atg12 and Bcl-2 interaction in GM-induced cochleotoxicity. As a result, the apoptotic function of Atg12 is inhibited, reflecting an increase in the number of hair cells. In summary, these results
A Input

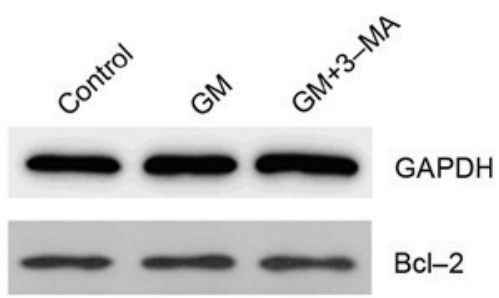

B IP:anti-Atg12

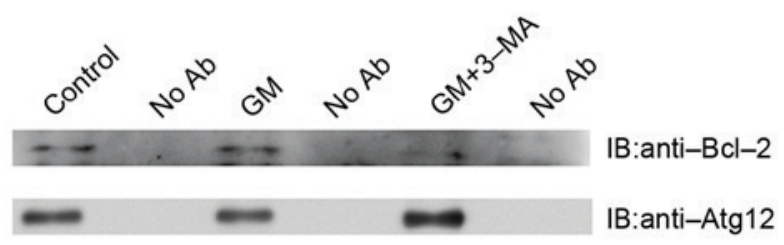

Figure 5. Co-immunoprecipitation of endogenous Atg12 with Bcl-2 in cochleae. (A) Representative western blot images of protein expression levels of Bcl-2. No significant differences were observed between all three groups. (B) IP result indicated that Bcl-2 interacts with Atg12 in the GM treated group, whereas in the $\mathrm{GM}+3$-MA treated group, Bcl-2 was not detected. GM, gentamicin; 3-MA, 3-methyladenine; Atg12, autophagy-related protein 12; Bcl-2, B-cell lymphoma 2; IP, immunoprecipitation.

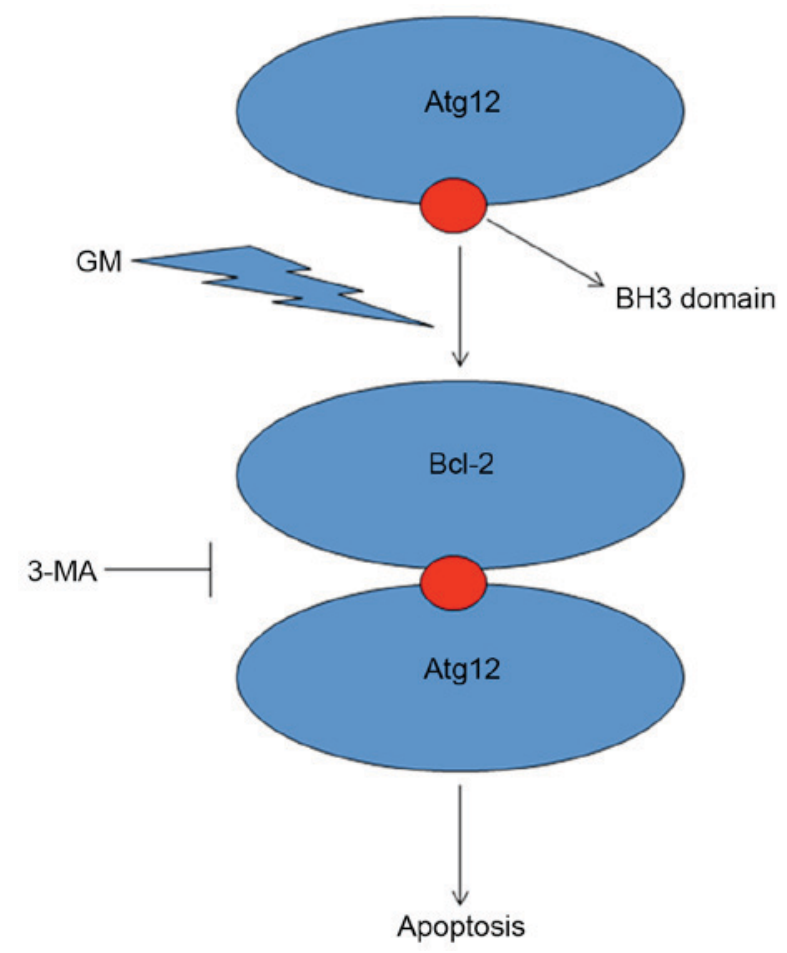

Figure 6. Schematic diagram of the interaction between Atg12 and Bcl-2. Atg12 contains a region with sequence similar to the to BH3-domain that is required for its interaction with Bcl-2 to induce apoptosis. Following GM treatment, Atg12 may associate with Bcl-2. When the autophagy inhibitor 3-MA is used, the Atg12-Bcl-2 interaction is blocked, inhibiting apoptosis. BH3, B cell lymphoma-2 homology 3-domain; GM, gentamicin; Bcl-2, B cell lymphoma-2; Atg12, autophagy-related protein 12 .

suggested that following GM-induced cochleotoxicity, Atg12 and $\mathrm{Bcl}-2$ interact to promote cochlear apoptosis, and 3-MA rescues this effect by inhibiting the Atg12-Bcl-2 complex. 


\section{Discussion}

Aminoglycosides, including GM, are primarily used for the treatment of aerobic systemic infection caused by gram-negative bacilli (26). However, GM treatment may induce hearing loss and balance disorders by damaging IHC structure in the cochlea (27). The clinical use of aminoglycosides including GM is limited due to their potential ototoxicity and nephrotoxicity (28). In recent years, a variety of cephalosporins and quinolones have been widely used in clinics because of their longer post-antibiotic effects against Pseudomonas aeruginosa, Klebsiella pneumoniae and Escherichia coli; however, aminoglycosides are used for the treatment of severe infections from aerobic gram-negative bacillus, including meningitis, trauma, burns and respiratory tract, urinary tract, skin soft tissue, joint and gastrointestinal tract infections (29). Previous studies have reported that the GM-induced hearing loss is associated with damage of cilia and hair cells $(1,30)$.

An important goal of preventing the ototoxicity of GM is to protect the hair cells from apoptosis; a well-known primary death mode following ototoxicity (7). Numerous drugs have been demonstrated to reduce apoptosis induced by GM ototoxicity (31-33), and apoptosis has been reported to be closely associated with autophagy (8). Notably, autophagy may cause cells to survive or may lead to cell death, although it is unclear which factors influence these transitional states $(14,34)$. Recent research on autophagy and the underlying mechanisms has developed; however, little is understood about the regulation and physiology of autophagy in cochlear tissue. The present study investigated GM-induced hair cell loss in neonatal mouse cochleae culture and demonstrated evidence of autophagy. Notably, it was revealed that inhibition of autophagy may influence the ototoxicity induced by GM.

The experiments in the present study were conducted in an in vitro model, where cultures of the organ of Corti were incubated with GM to induce cell stress injury that leads to hair cells death. This model has been used and characterized in numerous studies (35-37). The present study demonstrated the occurrence of autophagy in this model using multiple techniques, including examination of autophagosomes by electron microscopy, immunofluorescence and immunoblot analysis of LC3-II formation. In the current study, it was demonstrated that autophagic expression was increased following GM treatment; protein expression levels of the autophagy marker LC3 were upregulated.

Numerous previous studies have suggested that the interaction between autophagy and apoptosis extends to "core machinery' proteins, which serve as essential components of one signaling pathway and regulators of the other $(34,38,39)$. Another important autophagy regulator identified in the present study was Bcl-2. Bcl-2 is well recognized as an anti-apoptotic protein. In previous studies, Bcl-2 has been indicated to inhibit autophagy by binding to Beclin-1 (40-42). Bcl-2 binding to Beclin-1 may disrupt a protein complex (involving Beclin-1, ultra violet radiation resistance-associated gene protein, serine/threonine-protein kinase VPS15 and others) that is critical to vesicle nucleation, the start point of autophagosome formation (43). In certain cells, Atg12 is crucial for apoptosis, in addition to serving a role in autophagy (39). The autophagic function of Atg12 does not necessarily interfere with its role in apoptosis; the dual nature of these proteins suggests that interaction between the two signaling pathways is important for the activation and inhibition of apoptosis.

Atg12 contains a region with a sequence similar to Bcl-2 homology 3-domain that is required for its interaction with $\mathrm{Bcl}-2$ to induce apoptosis (18). Co-immunoprecipitation studies have indicated that Atg12 interacts with Bcl-2 in the cochlea. In conclusion, the results of the present study suggested that Atg12 has an apoptotic function via binding to Bcl-2 (Fig. 6). This binding may subsequently function as a molecular switch to activate apoptosis. These results implicate Atg12 as a potential therapeutic target for the treatment of GM-induced cochlear hair loss.

\section{Acknowledgements}

The present study was supported by the Nanjing Science and Technology Development Foundation (grant no. 201303003) and the Nanjing Medical Science and Technique Development Foundation (grant no. QRX11079). The authors would like to thank Professor Junfeng Zhang and Lei Dong from the State Key Laboratory of Pharmaceutical Biotechnology of Nanjing University (Nanjing, China), for providing technical assistance.

\section{References}

1. Bertolaso L, Bindini D, Previati M, Falgione D, Lanzoni I, Parmeggiani A, Vitali C, Corbacella E, Capitani S and Martini A: Gentamicin-induced cytotoxicity involves protein kinase $\mathrm{C}$ activation, glutathione extrusion and malondialdehyde production in an immortalized cell line from the organ of corti. Audiol Neurootol 8: 38-48, 2003.

2. Nordang L and Anniko M: Nitro-L-arginine methyl ester: A potential protector against gentamicin ototoxicity. Acta Otolaryngol 125: 1033-1038, 2005.

3. Wargo KA and Edwards JD: Aminoglycoside-induced nephrotoxicity. J Pharm Pract 27: 573-577, 2014.

4. Hahn H, Salt AN, Schumacher U and Plontke SK: Gentamicin concentration gradients in scala tympani perilymph following systemic applications. Audiol Neurootol 18: 383-391, 2013.

5. Salt AN, King EB, Hartsock JJ, Gill RM and O'Leary SJ: Marker entry into vestibular perilymph via the stapes following applications to the round window niche of guinea pigs. Hear Res 283: 14-23, 2012.

6. King EB, Salt AN, Eastwood HT and O'Leary SJ: Direct entry of gadolinium into the vestibule following intratympanic applications in Guinea pigs and the influence of cochlear implantation. J Assoc Res Otolaryngol 12: 741-751, 2011.

7. Forge A and Li L: Apoptotic death of hair cells in mammalian vestibular sensory epithelia. Hear Res 139: 97-115, 2000.

8. Marino G, Niso-Santano M, Baehrecke EH and Kroemer G: Self-consumption: The interplay of autophagy and apoptosis. Nat Rev Mol Cell Biol 15: 81-94, 2014.

9. Deter RL, Baudhuin P and De Duve C: Participation of lysosomes in cellular autophagy induced in rat liver by glucagon. J Cell Biol 35: C11-C16, 1967.

10. Klionsky DJ: Autophagy: From phenomenology to molecular understanding in less than a decade. Nat Rev Mol Cell Biol 8: 931-937, 2007.

11. Suzuki K and Ohsumi Y: Molecular machinery of autophagosome formation in yeast, Saccharomyces cerevisiae. FEBS Lett 581: 2156-2161, 2007.

12. Periyasamy-Thandavan S, Jiang M, Wei Q, Smith R, Yin XM and Dong Z: Autophagy is cytoprotective during cisplatin injury of renal proximal tubular cells. Kidney Int 74: 631-640, 2008.

13. Shintani T and Klionsky DJ: Autophagy in health and disease: A double-edged sword. Science 306: 990-995, 2004.

14. Levine B and Yuan J: Autophagy in cell death: An innocent convict? J Clin Invest 115: 2679-2688, 2005.

15. Reggiori F and Klionsky DJ: Autophagy in the eukaryotic cell. Eukaryot Cell 1: 11-21, 2002 
16. Tanida I, Ueno $\mathrm{T}$ and Kominami E: LC3 conjugation system in mammalian autophagy. Int J Biochem Cell Biol 36: 2503-2518, 2004.

17. Kabeya Y, Mizushima N, Ueno T, Yamamoto A, Kirisako T, Noda T, Kominami E, Ohsumi Y and Yoshimori T: LC3, a mammalian homologue of yeast Apg8p, is localized in autophagosome membranes after processing. Embo J 19: 5720-5728, 2000.

18. Klionsky DJ, Abdalla FC, Abeliovich H, Abraham RT, Acevedo-Arozena A, Adeli K, Agholme L, Agnello M, Agostinis P, Aguirre-Ghiso JA, et al: Guidelines for the use and interpretation of assays for monitoring autophagy. Autophagy 8: 445-544, 2012.

19. Klionsky DJ, Cregg JM, Dunn WA Jr, Emr SD, Sakai Y, Sandoval IV, Sibirny A, Subramani S, Thumm M, Veenhuis M and Ohsumi Y: A unified nomenclature for yeast autophagy-related genes. Dev Cell 5: 539-545, 2003.

20. Lautermann J, Dehne N, Schacht J and Jahnke K: Aminoglycoside- and cisplatin-ototoxicity: From basic science to clinics. Laryngorhinootologie 83: 317-323, 2004 (In German).

21. Chi le NU, Tabuchi K, Nakamagoe M,Nakayama M, Nishimura B and Hara A: Ceramide/sphingomyelin cycle involvement in gentamicin-induced cochlear hair cell death. Arch Toxicol 89 415-421, 2015.

22. Ding D, Stracher A and Salvi RJ: Leupeptin protects cochlear and vestibular hair cells from gentamicin ototoxicity. Hear Res 164: 115-126, 2002.

23. Ding DL, Wang J, Salvi R, Henderson D, Hu BH, McFadden SL and Mueller M: Selective loss of inner hair cells and type-I ganglion neurons in carboplatin-treated chinchillas. Mechanisms of damage and protection. Ann N Y Acad Sci 884: 152-170, 1999.

24. Hofstetter P, Ding D, Powers N and Salvi RJ: Quantitative relationship of carboplatin dose to magnitude of inner and outer hair cell loss and the reduction in distortion product otoacoustic emission amplitude in chinchillas. Hear Res 112: 199-215, 1997.

25. Morell M, Lenoir M, Shadwick RE, Jauniaux T, Dabin W, Begeman L, Ferreira M, Maestre I, Degollada E, Hernandez-Milian G, et al: Ultrastructure of the Odontocete organ of Corti: Scanning and transmission electron microscopy. J Comp Neurol 523: 431-448, 2015.

26. Gomółka M and Niemczyk S: How to safely and effectively administer aminoglycoside antibiotics. Pol Merkur Lekarski 36: 225-258, 2014 (In Polish).

27. Theopold HM: Comparative surface studies of ototoxic effects of various aminoglycoside antibiotics on the organ of Corti in the guinea pig. A scanning electron microscopic study. Acta Otolaryngol 84: 57-64, 1977.

28. Forge A and Schacht J: Aminoglycoside antibiotics. Audiol Neurootol 5: 3-22, 2000.

29. Chen C, Chen Y, Wu P and Chen B: Update on new medicinal applications of gentamicin: Evidence-based review. J Formos Med Assoc 113: 72-82, 2014
30. Maudonnet EN, de Oliveira JA, Rossato $M$ and Hyppolito MA: Gentamicin attenuates gentamicin-induced ototoxicity-self-protection. Drug Chem Toxicol 31: 11-25, 2008.

31. Du XF, Song JJ, Hong S and Kim J: Ethanol extract of Piper longum L. attenuates gentamicin-induced hair cell loss in neonatal cochlea cultures. Pharmazie 67: 559-563, 2012.

32. Choung YH, Kim SW, Tian C, Min JY, Lee HK, Park SN, Lee JB and Park K: Korean red ginseng prevents gentamicin-induced hearing loss in rats. Laryngoscope 121: 1294-1302, 2011.

33. Chang J, Jung HH, Yang JY, Choi J, Im GJ and Chae SW: Protective role of antidiabetic drug metformin against gentamicin induced apoptosis in auditory cell line. Hear Res 282: 92-96, 2011.

34. Maiuri MC, Zalckvar E, Kimchi A and Kroemer G: Self-eating and self-killing: Crosstalk between autophagy and apoptosis. Nat Rev Mol Cell Biol 8: 741-752, 2007.

35. Bas E, Van De Water TR, Gupta C, Dinh J, Vu L, Martínez-Soriano F, Láinez JM and Marco J: Efficacy of three drugs for protecting against gentamicin-induced hair cell and hearing losses. Br J Pharmacol 166: 1888-1904, 2012.

36. Yadav MK, Choi J and Song JJ: Protective effect of hexane and ethanol extract of piper longum L. On gentamicin-induced hair cell loss in neonatal cultures. Clin Exp Otorhinolaryngol 7: 13-18, 2014

37. Choung YH, Taura A, Pak K, Choi SJ, Masuda M and Ryan AF: Generation of highly-reactive oxygen species is closely related to hair cell damage in rat organ of Corti treated with gentamicin. Neuroscience 161: 214-226, 2009.

38. Eisenberg-Lerner A, Bialik S, Simon HU and Kimchi A: Life and death partners: Apoptosis, autophagy and the cross-talk between them. Cell Death Differ 16: 966-975, 2009.

39. Rubinstein AD, Eisenstein M, Ber Y, Bialik S and Kimchi A: The autophagy protein Atg12 associates with antiapoptotic Bcl-2 family members to promote mitochondrial apoptosis. Mol Cell 44: 698-709, 2011.

40. Erlich S, Mizrachy L, Segev O, Lindenboim L, Zmira O, Adi-Harel S, Hirsch JA, Stein R and Pinkas-Kramarski R: Differential interactions between Beclin 1 and $\mathrm{Bcl}-2$ family members. Autophagy 3: 561-568, 2007.

41. Germain M, Nguyen AP, Le Grand JN, Arbour N, Vanderluit JL, Park DS, Opferman JT and Slack RS: MCL-1 is a stress sensor that regulates autophagy in a developmentally regulated manner. Embo J 30: 395-407, 2011.

42. Pattingre S, Tassa A, Qu X, Garuti R, Liang XH, Mizushima N, Packer M, Schneider MD and Levine B: Bcl-2 antiapoptotic proteins inhibit Beclin 1-dependent autophagy. Cell 122: 927-939, 2005.

43. Pattingre S and Levine B: Bcl-2 inhibition of autophagy: A new route to cancer? Cancer Res 66: 2885-2889, 2006. 